\title{
Low-Power Laser Irradiation (LPLI): A Clinical Point of View on a Promising Strategy to Improve Liver Regeneration
}

\author{
Tiago Gomes Araújo ${ }^{1,2^{*}}$, Alexandre Gabarra Oliveira ${ }^{1,3}$, Antonio R. Franchi Teixeira ${ }^{4}$ \\ ${ }^{1}$ Department of Internal Medicine, State University of Campinas (UNICAMP), Campinas, SP, Brazil \\ ${ }^{2}$ Department of Physiology and Pharmacology, Federal University of Pernambuco (UFPE), Recife, PE, Brazil \\ ${ }^{3}$ Institute of Biosciences Institute, São Paulo State University (UNESP), Rio Claro, SP, Brazil \\ ${ }^{4}$ Campinas Liver Institute, Campinas, São Paulo, Brazil
}

\section{*Correspondence to \\ Tiago Gomes Araújo, PhD; \\ Department of Internal Medicine, \\ Faculty of Medical Science/ \\ State University of Campinas, \\ Cidade Universitária Zeferino Vaz, \\ Campinas-SP, Brazil, 13081-970, \\ Fax: +55-19-35218950; \\ Email: tigaraujo@hotmail.com}

Published online 17 September 2018

\begin{abstract}
The capacity of the liver to regenerate is an important clinical issue after major hepatectomies and makes the difference between life and death in some cases of post-operative malfunction when the liver remnant is too small or has an impaired regenerative capacity. Several approaches have been tested to stimulate hepatic regeneration after post-operative hepatic failure syndrome; however, they have produced controversial results. A quick, simple, and harmless method that can be used intraoperatively and capable of promoting an increased regenerative capacity of the remaining liver would be very welcome. Thus, based on the data in the literature, we presented low-power laser irradiation (LPLI) as a quick, simple, and harmless method to improve liver regeneration after major hepatectomies. This article highlights the current evidence about the effects of LPLI on liver regeneration, and also suggests laser therapy as an important tool for regenerative stimulation in clinical practice.
\end{abstract}

Keywords: Clinical; Liver regeneration; Laser treatment; Hepatectomy; Liver resection; Lowpower laser irradiation; LPLI.

\section{Introduction}

The regeneration capacity of the liver has been known since the Classical Era. It is reported by Hesiod in his Theogony, written in the eighth century BC, that the titan Prometheus, a defender of humanity who was known for his great intelligence, challenged Zeus by stealing fire from Olympus and providing it to mortals. Zeus punished Prometheus for this crime, leaving him chained to a rock on the top of the Caucasus Mountain for eternity while a big eagle would eat part of his liver every day, which would grow again the next day. Despite the immortality of Prometheus, the regenerative process of the liver, described in this passage from Greek mythology, is the most famous historical reference to the hepatic regeneration capacity in literature.

Biologically, the liver is an organ of complex metabolic functions. It receives blood efflux from the gut and the venous drainage of the pancreas and spleen. This anatomical particularity of continuous blood flow from the mesenteric region gives the liver some particular properties of supply. Indeed, it provides the specific biochemical characteristics to the hepatocyte, such as defense against toxic agents and a distinctive expression pattern of genes and proteins. Since the liver has paramount homeostatic functions in all species, the evolutionary process tried to preserve its maximum function. A remarkable characteristic of this organ is the ability to regenerate its functional mass without sacrificing the body homeostasis. This phenomenon is observed in all vertebrates, from fish to humans. In humans this remarkable regenerative capacity is very important in resectional surgery, since the liver is a common place of primary and secondary malignancies that must be resected.

\section{Clinical Relevance of a Hepatectomy}

Several benign and malignant diseases can affect the liver. For most of these tumors, resectional surgery is still the treatment of choice. Partial hepatectomy is the treatment chosen in many cases of hepatocellular carcinoma and liver metastases. Concerning hepatocellular carcinoma, many of these tumors appear as a result of cirrhosis and chronic hepatitis of various etiologies. In cases of primary liver cancer in patients with preserved liver function and the absence of a significant portal hypertension, liver resection may be the method of choice for the treatment, sometimes using the laparoscopic approach. In cases of metastatic disease, colorectal carcinoma is the most 
prevalent, and liver resection remains the most effective treatment for this condition.

Recently, liver resection surgery has experienced an impressive evolution. Using the recognized regenerative capacity of the remnant liver, several strategies have been described for the surgical treatment of primary and secondary organ malignancies. ${ }^{1-3}$ Also, more sophisticated and minimally invasive techniques, such as laparoscopy and robotic surgery, that were once considered impossible, have been successfully used in performing hepatectomies. In cases of hepatocarcinoma (HCC), resection of more than one liver sector is common nowadays. ${ }^{2,4}$ Compensated cirrhotic patients with HCC now undergo liver resection, even using laparoscopic techniques. In all cases, the liver remnant is expected to regenerate. Unfortunately, this does not always happen.

Elderly and steatotic livers patients may have an impaired capacity of recovery after a major hepatecomies. Concerning colorectal liver metastasis, the utilization of pre-operative chemotherapies based on oxaliplatin or irinotecan also may lead to some conditions that impair the capacity of liver regeneration (steatohepatitis and sinusoidal obstruction). ${ }^{5}$ Unfortunately, in some cases the regenerative capacity of the remnant liver is compromised, either by the use of chemotherapy or the presence of chronic inflammation and fibrosis. Postoperative hepatic failure is still a difficult condition to treat, since the remaining liver sometimes shows an unsatisfactory regeneration capacity. This means that the liver regeneration capacity is crucial after major hepatectomies and makes the difference between life and death in some cases when the liver remnant is very small or has an impaired regenerative capacity. Extended hepatectomy surgery carried out in 2 stages and preceded by maneuvers that stimulate liver regeneration is a recently developed strategy that has changed the resectability rate of hepatic tumors.

\section{The Current Clinical Practice to Prevent Post-operative Hepatic Failure Syndrome After a Hepatectomy}

In order to avoid the syndrome of liver failure in the post-operative period of a hepatectomy, various strategies have been used before carrying out a major hepatectomy. The first and most obvious and simple of these strategies is the correct selection of patients that should undergo surgery. Many patients with hepatocellular carcinoma as a result of chronic hepatitis or cirrhosis are not suitable for resection and are best treated by liver transplantation or ablative therapies such as arterial chemoembolization or percutaneous ablation (ethanol, microwaves, radiofrequency, electroporation). In the case of metastases, resection is the treatment with the highest cure rates, but many patients receive high doses of chemotherapy and show changes in the liver that affect its regenerative capacity in the post-operative period.

Several strategies have been tested in order to stimulate liver regeneration after hepatectomies. Since liver regeneration depends on the blood portal flow (rich in insulin, peptides, and hepatotrophic factors) that runs through the liver, pre-operatory portal vein embolization (PVE) deviates the portal flow to the contralateral lobe. The hemodynamic changes achieved by PVE lead to a blood portal overflow to the contralateral side, while maintaining the arterial blood supply in the affected lobe. ${ }^{6}$ This maneuver prevents necrosis of the effected lobe but induces hypertrophy of the contralateral lobe, increasing the function of the future remaining liver. There is evidence that the production of inflammatory cytokines, vessel regulators, and growth factors is involved in the hypertrophy process. ${ }^{3,6}$ What is theoretically achieved with PVE is an anticipation of the regenerative capacity of the remaining liver, which becomes stimulated pre-operatively. However, there are doubts about the possibility that PVE can offer an "extra" regenerative capacity in the remaining liver.

In some cases, PVE fails to induce hypertrophy of the contralateral lobe. This may be related to an unidentified regenerative dysfunction in the liver. In these cases, many health facilities understand that the patient should not undergo a hepatectomy, since liver failure in the postoperative period is very likely to occur. Working as a selection criterion, this strategy can prevent death in the post-operative period of a major hepatectomy. On the other hand, other authors argue that it is necessary to try other alternatives in such cases. ${ }^{3}$ The technique known as ALPPS (Associated Liver Partition and Portal vein ligation for Staged hepatectomy) has been described as an efficient alternative to induce growth of the remaining liver when PVE fails. ${ }^{3,7}$ ALPPS is a 2-stage surgical strategy in which, in the first procedure, the liver is subjected to a partition and portal ligation on the affected lobe. This maneuver is believed to completely disconnect both sides of the liver, and a more efficient portal flow deviation can improve regeneration of the remnant. After 7-14 days, it is expected a large hypertrophy in the contralateral lobe, then the patient is submitted to a second surgery to remove the affected lobe. However, this method requires 2 major surgeries, and consequently promotes an increased length of hospital stay and the risk of biliary fistulas and infections. ${ }^{7,8}$

Is portal flow the only way to stimulate liver regeneration? Regeneration of solid organs is a complex process that comes in waves. Firstly, ancient proto-oncogenes are stimulated by aggression stimuli like ischemia or trauma. Then, several molecular pathways are mobilized in order to induce inflammation. Many of these pathways are promiscuous and may induce regenerative pathways. Afterwards, RNA messages are sent to cellular structures to promote hypertrophy and/or hyperplasia. The cells then need to duplicate, which consumes much energy. ${ }^{9,10}$ Mitochondria are very important in this process, since they provide the necessary energy. ${ }^{11}$ Mitochondria have 
photon receptors and may respond to light stimulation. Light can also stimulate melatonin production in the central nervous system (CNS), and melatonin has a recognized capacity to stimulate regeneration in some tissues and organs. ${ }^{11,12}$ So the question is, does light have the potential to induce liver regeneration?

\section{New Road - the Low-Power Laser Irradiation}

Light is known to be an important factor for the homeostasis of many tissues and organs. Absorption and utilization of vitamins, hormonal circadian cycles, and control of wakefulness and sleep systems are examples of light-dependent homeostatic systems. The LASER (light amplification by stimulated emission of radiation) invention, allowed to obtain light with high power and in certain wavelengths, facilitating its application in biology and allowing a new field of research related to the biological effects of radiation on animals and humans. ${ }^{12}$ As a result, by the 1970s there appeared the first satisfactory results of the biological effects of lasers, as it was found that LPLI caused the stimulation of healing in surgical wounds. ${ }^{13}$ Following this line, further studies were conducted in this new field of research.

Currently, LPLI is used in clinical practice due to a very important physiological effect: the induction of cell proliferation. ${ }^{12,14}$ The stimulatory effect of LPLI on cell regeneration and consequent resolution of ischemic or mechanical problems in organs such as the skeletal muscle, heart, brain, and liver are well known. ${ }^{14}$ Furthermore, there is evidence that the gene or protein expressions of several growth factors, such as transforming growth factor- $\beta$ (TGF- $\beta$ ), vascular endothelial growth factor (VEGF), platelet-derived growth factor (PDGF), and insulinlike growth factor I (IGF-I), are elevated by LPLI. ${ }^{12,14}$ Thus, it is clear that through the increased expressions of these growth factors, LPLI is able to enhance cellular proliferation and differentiation.

Until recently, there were few lines of research related to the action of LPLI on liver regeneration (see Table 1 and Figure 1). First, it was recognized that there is a universal photo-biological mechanism of the light in the terminal enzymes of the respiratory chain in mitochondria from eukaryotic cells. ${ }^{11}$ In this regard, some groups have studied the effects of lasers on mitochondrial function. It is reported in the literature that lasers have the ability to induce conformational changes in the structure of cytochromes from hepatocytes, thereby increasing the production of adenosine triphosphate (ATP). ${ }^{15-17}$ In this line, an increase in energy metabolism in the liver of a hepatectomizated rat exposed to laser treatment was observed. ${ }^{15-17}$ In a recent study, Barbosa and colleagues observed that 24 hours after laser exposure have already observed an important increase in ATP production. ${ }^{18}$ In sum, these studies demonstrated that both high- and low-power lasers have the ability to increase the mitotic index and mitochondrial function in the remaining liver
Table 1. The Main Parameters of the Laser Used to Induce the Liver Regeneration

\begin{tabular}{lccc}
\hline Wavelength $(\mathbf{n m})$ & $\begin{array}{c}\text { Intensity } \\
(\mathbf{m W} / \mathbf{c m})\end{array}$ & $\begin{array}{c}\text { Irradiation } \\
\text { Time }(\mathbf{s})\end{array}$ & Reference(s) \\
\hline 590 & 50 & 300 & 15 \\
$410,470,512$, & 50 & 300 & 16 \\
590 and 630 & 50 & 150 & 18 \\
660 & 30 & 150 & 19 \\
660 & 5 & 60 & 20 \\
810 & 65 & 900 & 21,23 \\
632.8 & & & \\
\hline
\end{tabular}

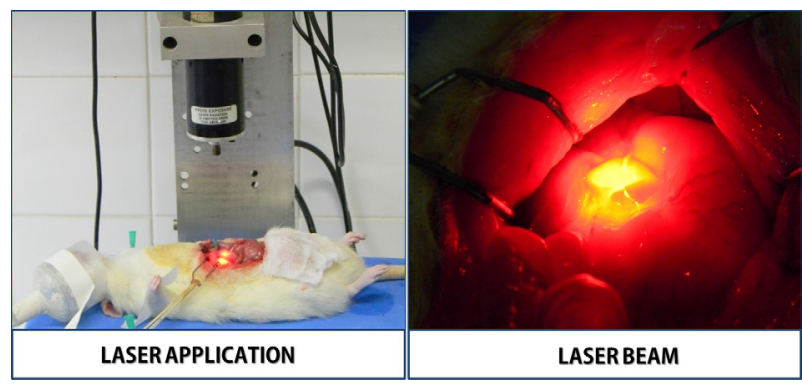

Figure 1. Details of the Laser Application Procedure. These images was based from previous studies. ${ }^{21,23}$

after different levels of partial resection (70\% and 90\%) in rats. ${ }^{15-19}$

In addition, Oron et $\mathrm{al}^{20}$ in analyzing other parameters, demonstrated the effects of LPLI on liver regeneration in rats subjected to a $70 \%$ hepatectomy. Two days after the surgery and laser exposure, it was observed that the remaining liver had increased the newly formed blood vessels determined by 5 -Bromo-2'deoxyuridine (BrdU immunostaining), as well as, had augmented the number of bone-marrow-derived mesenchymal stem cells (MSCs) determined by c-kit immunostaining. Thus, it was concluded that LPLI causes a significant improvement in hepatic regeneration through an increase in the formation of new hepatocytes, MSCs, and angiogenesis in the remaining liver. ${ }^{20}$

More recently, our group conducted further studies related to the effect of laser treatment in animals subjected to a partial hepatectomy. Using immunoblotting and immunostaining approaches, we investigated the main molecular mechanisms involved in liver regeneration of partially hepatectomized (70\%) rats exposed to LPLI. Several studies have demonstrated that a hepatotrophic factor called hepatocyte growth factor (HGF), which binds to a specific receptor (c-Met) and comes from the family of tyrosine kinases, has an important role in the liver regeneration mechanism. ${ }^{9,10}$ The progression of the cell cycle can be regulated through activation or expression of specific proteins. Consequently, well-known intracellular signaling pathways such as Ras/Raf/MEK/Erk, PI3K/Akt/ eIF4E, PI3K/Akt/eNOS, and PLC-gamma/PKC may be 
involved in the regenerative effect of the laser in various tissues. ${ }^{12,14}$ This data led us to hypothesize the effect of the laser on the regenerative molecular pathway HGF $\rightarrow$ Met $\rightarrow$ Akt and Erk 1/2. Then, 2 days after partial hepatectomy surgery and exposure to the laser in rats, we studied the remaining livers. Our results showed that LPLI can improve liver regeneration, as evidenced by increasing HGF protein expression and the phosphorylation levels of Met, Akt, and Erk 1/2, along with higher levels of PCNA and Ki-67 (established proliferating markers) in the remnant liver of partially hepatectomized rats. ${ }^{21}$

Once we demonstrated that LPLI was able to improve liver regeneration in partially hepatectomized rats, we also tried to demonstrate the same effects in elderly rats subjected to the same surgery. This is an important target for this adjuvant therapy, since studies in humans have demonstrated that those over 65 years having three to 5-fold higher fatal outcomes of liver disease when compared with those under 45 years old. ${ }^{22}$ Despite the remarkable results observed in young hepatectomized animals, LPLI failed to improve liver regeneration in elderly rats, as evidenced by the lack of increase in the phosphorylation levels of Met, Akt, and ERK1/2 in the liver. Also, we did not observe any significant improvement in HGF and PCNA protein expression. ${ }^{23}$ Taken together, the results of this study indicate that LPLI cannot induce any improvement in the hepatic regeneration process for at least 48 hours after $70 \%$ resection, which excludes LPLI as an auxiliary therapy in elderly hepatectomized rats. ${ }^{23}$

\section{Conclusion}

In the clinical context of a major hepatectomy, there is an ongoing need to prevent the syndrome of post-operative liver failure by dysfunction of the remaining liver. Techniques such as pre- and post-conditioning (ischemic, pharmacological, or related to hepatic hemodynamics) have been used with little convincing results to date. The vast majority of techniques that aim to advance or improve the regenerative capacity of the liver remnant require prior planning (e.g. PVE). When these pre-operative maneuvers are not performed because they are seen as superfluous or unnecessary is common the surgeon to come across in a very difficult intraoperative decisions in which the continuation with surgery means leaving a dangerously small liver remnant. When the decision is to proceed with the surgery, it is observed that liver failure may occur in the first post-operative days, which consequently causes encephalopathy and coagulopathy, ending in a fatal outcome in the majority of cases. Only intensive care support can be offered to patients who are in this situation because there is no way to improve the regenerative capacity of the remaining liver at that point. There are doubts about whether low-power laser stimulation in tissues where there are nested neoplastic cells could stimulate their replication, leading to disease progression. In clinical practice, it is known that the use of intraoperative ultrasound directly on the remaining liver is the most sensitive method for the detection of small tumors from $2-3 \mathrm{~mm}$ in diameter. Fortunately most of reference services in liver surgery have such equipment as routine. Therefore, an idea would be to conduct intraoperative ultrasonography in patients' candidate to receive stimulation of LPLI. A quick, simple, and harmless method that can be used intraoperatively and capable of promoting an increased regenerative capacity of the remaining liver would be very welcome.

Liver regeneration is a phenomenon related to a variety of different mechanisms. Portal flow deviation is the most utilized strategy to increase regeneration of the liver remnant prior to (e.g. PVE) or during (e.g. ALPSS) surgery, but other strategies might also be useful in the clinical setting. LPLI may be a useful and harmless intraoperative tool to improve the regenerative capacity of a small liver remnant. In sum, laser therapy to induce the improvement of hepatic regeneration is composed of a variety of mechanisms. In this sense, according to data from the literature and our experimental data, we can provide an overview of LPLI effects on liver regeneration (Figure 2). LPLI induces an increase in the mitotic index and mitochondrial function and augments the formation of new hepatocytes and MSCs along with angiogenesis in the remaining liver. Indeed, LPLI can improve liver regeneration, as documented by increasing HGF protein expression and the phosphorylation levels of Met, Akt, and Erk 1/2, along with higher levels of PCNA and Ki67. Therefore, in the near future, laser therapy could be an important tool for regenerative stimulation of the remaining liver during a large liver resection.

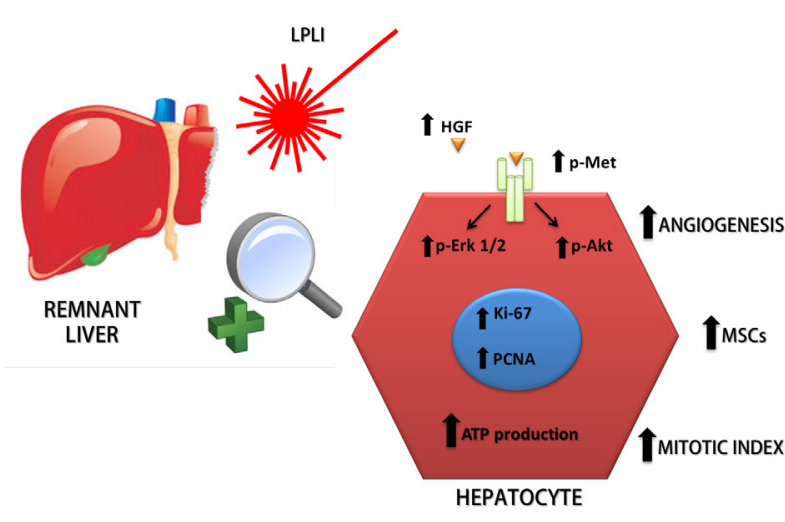

Figure 2. LPLI-Induced Mechanisms Involved in the Enhancement of Hepatic Regeneration. The figure shows the effects of LPLI on hepatic regeneration. LPLI causes an increase in the mitotic index and mitochondrial function and augments the formation of new hepatocytes, MSCs, and angiogenesis in the remaining liver. Indeed, LPLI can improve liver regeneration, as evidenced by the enhanced regenerative molecular pathway HGF $\rightarrow$ Met $\rightarrow$ Akt and Erk 1/2, along with higher levels of PCNA and Ki-67. LPLI: Low-Power Laser Irradiation, MSCs: bone-marrow-derived mesenchymal stem cells, HGF: hepatocyte growth factor, PCNA: proliferating cell nuclear antigen. 


\section{Ethical Considerations}

Not applicable.

\section{Conflict of Interests}

The authors declare no conflict of interest.

\section{Acknowledgments}

This work was supported by grants from the Coordenação de Aperfeiçoamento de Pessoal de Nível Superior/PNPD (Capes/PNPD) and Conselho Nacional de Pesquisa (CNPq). No potential conflicts of interest relevant to this article were reported.

\section{References}

1. Morise Z, Kawabe N, Tomishige H, et al. Recent advances in liver resection for hepatocellular carcinoma. Front Surg. 2014;1:21. doi:10.3389/fsurg.2014.00021

2. Kwon YJ, Lee KG, Choi D. Clinical implications of advances in liver regeneration. Clin Mol Hepatol. 2015;21(1):7-13. doi:10.3350/cmh.2015.21.1.7

3. Dimitroulis D, Tsaparas P, Valsami S, et al. Indications, limitations and maneuvers to enable extended hepatectomy: current trends. World J Gastroenterol. 2014;20(24):78877893. doi:10.3748/wjg.v20.i24.7887

4. Morise Z, Sugioka A, Kawabe N, et al. Pure laparoscopic hepatectomy for hepatocellular carcinoma patients with severe liver cirrhosis. Asian J Endosc Surg. 2011;4(3):143146. doi:10.1111/j.1758-5910.2011.00081.x

5. Pessaux P, Chenard MP, Bachellier P, Jaeck D. Consequences of chemotherapy on resection of colorectal liver metastases. J Visc Surg. 2010;147(4):e193-201. doi:10.1016/j. jviscsurg.2010.06.004

6. Vyas S, Markar S, Partelli S, et al. Portal vein embolization and ligation for extended hepatectomy. Indian J Surg Oncol. 2014;5(1):30-42. doi:10.1007/s13193-013-0279-y

7. Vennarecci G, Laurenzi A, Santoro R, Colasanti M, Lepiane P, Ettorre GM. The ALPPS procedure: a surgical option for hepatocellular carcinoma with major vascular invasion. World J Surg. 2014;38(6):1498-1503. doi:10.1007/s00268013-2296-y

8. Zhang GQ, Zhang ZW, Lau WY, Chen XP. Associating liver partition and portal vein ligation for staged hepatectomy (ALPPS): a new strategy to increase resectability in liver surgery. Int J Surg. 2014;12(5):437-441. doi:10.1016/j. ijsu.2014.03.009

9. Michalopoulos GK. Liver regeneration. J Cell Physiol. 2007;213(2):286-300. doi:10.1002/jcp.21172
10. Michalopoulos GK. Liver regeneration after partial hepatectomy: critical analysis of mechanistic dilemmas. Am J Pathol. 2010;176(1):2-13. doi:10.2353/ajpath.2010.090675

11. Karu TI, Kolyakov SF. Exact action spectra for cellular responses relevant to phototherapy. Photomed Laser Surg. 2005;23(4):355-361. doi:10.1089/pho.2005.23.355

12. Hamblin MR, Huang Y. Handbook of Photomedicine. Boca Raton: CRC Press; 2014.

13. Mester E, Szende B, Spiry T, Scher A. Stimulation of wound healing by laser rays. Acta Chir Acad Sci Hung. 1972;13(3):315-324.

14. Gao X, Xing D. Molecular mechanisms of cell proliferation induced by low power laser irradiation. J Biomed Sci. 2009;16:4. doi:10.1186/1423-0127-16-4

15. de Castro e Silva Junior O, Zucoloto S, Menegazzo LA, Granato RG, Marcassa LG, Bagnato VS. Laser enhancement in hepatic regeneration for partially hepatectomized rats. Lasers Surg Med. 2001;29(1):73-77. doi:10.1002/1sm.1089

16. Castro-e-Silva O Jr, Zucoloto S, Marcassa LG, et al. Spectral response for laser enhancement in hepatic regeneration for hepatectomized rats. Lasers Surg Med. 2003;32(1):50-53. doi:10.1002/lsm.10141

17. Castro-e-Silva O Jr, Silveira MRG. The liver and light. A precise combination, from mythology to medicine. Medicina (Ribeirão Preto). 2013;46:318-321.

18. Barbosa AJ, Santana AC, Castro e Silva T, et al. Effect of laser on the remnant liver after the first 24 hours following 70\% hepatectomy in rats. Acta Cir Bras. 2011;26(6):470474.

19. Oliveira AF, Silva TC, Sankarankutty AK, et al. The effect of laser on remanescent liver tissue after $90 \%$ hepatectomy in rats. Acta Cir Bras. 2006;21 Suppl 1:29-32.

20. Oron U, Maltz L, Tuby H, Sorin V, Czerniak A. Enhanced liver regeneration following acute hepatectomy by lowlevel laser therapy. Photomed Laser Surg. 2010;28(5):675678. doi:10.1089/pho.2009.2756

21. Araujo TG, de Oliveira AG, Tobar N, et al. Liver regeneration following partial hepatectomy is improved by enhancing the HGF/Met axis and Akt and Erk pathways after low-power laser irradiation in rats. Lasers Med Sci. 2013;28(6):1511-1517. doi:10.1007/s10103-013-1264-y

22. Regev A, Schiff ER. Liver disease in the elderly. Gastroenterol Clin North Am. 2001;30(2):547-563. doi:10.1016/S08898553(05)70195-3

23. Araujo TG, Oliveira AG, Tobar N, et al. Low-power laser irradiation fails to improve liver regeneration in elderly rats at $48 \mathrm{~h}$ after $70 \%$ resection. Lasers Med Sci. 2015;30(7):2003-2008. doi:10.1007/s10103-014-1598-0 